\title{
Koiter's Shell Theory from the Perspective of Three-dimensional Nonlinear Elasticity
}

\section{David J. Steigmann}

\begin{abstract}
Koiter's shell model is derived systematically from nonlinear elasticity theory, and shown to furnish the leading-order model for small thickness when the bending and stretching energies are of the same order of magnitude. An extension of Koiter's model to finite midsurface strain emerges when stretching effects are dominant.
\end{abstract}

Keywords Shell theory $\cdot$ Nonlinear elasticity

Mathematics Subject Classification 74B20 - 74K25

\section{Introduction}

Koiter's nonlinear shell theory, which purports to be valid for isotropic shells undergoing finite deformations with small strains, enjoys a unique status. This stems in large part from the extensive assessment of it in recent years, on the part of Ciarlet and his school, as an approximation to elasticity theory for thin bodies. A large body of evidence is given in [1] in support of the contention that the Koiter model furnishes the 'best' all-around theory, despite the fact that it has not been obtained either as a gamma limit or an asymptotic limit of the three-dimensional theory. The important features of Koiter's theory are associated with the areal strain-energy density (see [1], p. 156 and [2], p. 30)

$$
W={ }_{2}^{1} h \boldsymbol{\epsilon} \cdot \mathcal{D}[\boldsymbol{\epsilon}]+{ }_{24}^{1} h^{3} \rho \cdot \mathcal{D}[\boldsymbol{\rho}],
$$

where $\mathcal{D}$ is the positive-definite tensor of linear plane-stress elastic moduli, $h$ is the thickness of the shell prior to deformation, and $\epsilon$ and $\rho$ respectively are surface tensors that characterize the change in metric and curvature of the shell midsurface induced by deformation. Accordingly, by the fundamental theorem of surface theory [1], the energy vanishes for rigid-body motions of the midsurface and is positive definite for any non-rigid motion.

\footnotetext{
D.J. Steigmann ( $\varangle)$

Department of Mechanical Engineering, University of California, Berkeley, CA 94720, USA

e-mail: steigman@me.berkeley.edu
} 
In the present work we revisit Koiter's theory for the purpose of providing a pedagogically useful derivation of the model together with an understanding of its position vis $a$ vis nonlinear elasticity theory. Although we do not use the methods of gamma convergence theory explicitly, the reader will recognize our leading-order energy, pertaining to states in which the bending and stretching energies are of the same order, as formally coinciding with the gamma (or asymptotic) limit. Remarkably, this limit is exactly Koiter's expression for the energy [2]. Our procedure may be viewed as an update of that used by Koiter in his original treatment [3]. In particular we seek a small-thickness estimate, for a given deformation, of the potential energy of an edge-loaded shell.

The present work draws on a wide range of ideas developed over the history of shell theory. For this reason a number of pre-requisite facts are quoted from various sources, rather than developed explicitly. Standard bold face notation is used for vectors and tensors and indices to denote their components. Latin indices take values in $\{1,2,3\}$; Greek in $\{1,2\}$. The latter are associated with surface coordinates and attendant vector and tensor components. A dot between bold symbols is used to denote the standard Euclidean inner product; if $\mathbf{A}_{1}$ and $\mathbf{A}_{2}$ are second-order tensors, then their inner product is $\mathbf{A}_{1} \cdot \mathbf{A}_{2}=\operatorname{tr}\left(\mathbf{A}_{1} \mathbf{A}_{2}^{t}\right)$, where $\operatorname{tr}(\cdot)$ is the trace and the superscript ${ }^{t}$ is used to denote the transpose. The associated norm is $|\mathbf{A}|=\sqrt{\mathbf{A} \cdot \mathbf{A}}$. The notation $\otimes$ identifies the standard tensor product of vectors. The symbols Div and $D$ are used for the three-dimensional divergence and gradient operators, while div and $\nabla$ are reserved for their two-dimensional counterparts on a curved surface. We use a combination of direct notation and the indicial tensor notation preferred by shell theorists. Regarding the latter, useful background material, together with a summary of the requisite differential geometry of surfaces, may be found in [1,2]. Commas followed by subscripts are used to denote partial derivatives with respect to convected surface coordinates, while vertical strokes are used for covariant derivatives on the reference surface.

Superposed tildes are used to denote three-dimensional fields whereas variables appearing without the tilde are the restrictions of these fields to a midsurface $\Omega$ embedded in the three-dimensional shell-like body. The body itself occupies the volume $\kappa=$ $\Omega \times[-h / 2, h / 2]$. Our basic assumption is that the plate is thin in the sense that $h / l \ll 1$, where $l$ is any characteristic length associated with the geometry of $\Omega$. This is taken to be the smaller of the minimum radius of curvature of the shell or the minimum spanwise dimension. To ease the notation, we assume that all length scales have been non-dimensionalized by $l$ a priori. We then have $h \ll 1$, where $h$ is now the dimensionless thickness, and we seek a model for the elastic shell valid to order $h^{3}$.

\section{Three-dimensional Theory}

In a purely mechanical setting the Piola stress of the three-dimensional theory is given by

$$
\tilde{\mathbf{P}}=\mathcal{W}_{\tilde{\mathbf{F}}},
$$

the derivative with respect to the deformation gradient $\tilde{\mathbf{F}}$ of the strain energy, $\mathcal{W}(\tilde{\mathbf{F}})$, per unit reference volume. This is assumed, for the sake of simplicity, not to depend explicitly on the position $\mathbf{X}$ of a material point in the reference $\kappa$. Thus we confine attention to materials with uniform properties. The force per unit area transmitted across a surface in the reference configuration with unit normal $\mathbf{N}$ is

$$
\tilde{\mathbf{t}}=\tilde{\mathbf{P}} \mathbf{N}
$$


It is well known that this, together with the equilibrium equation

$$
\operatorname{Div} \tilde{\mathbf{P}}=\mathbf{0} \text {, }
$$

are the natural boundary condition and Euler equation for energy-minimizing deformations under conditions of conservative loading without body force, holding on a subset of $\partial \kappa$ and in $\kappa$ respectively.

We seek an expression for the term $E$ in the expansion

$$
\mathcal{E}=E+o\left(h^{3}\right)
$$

of the potential energy

$$
\mathcal{E}=\int_{\kappa} \mathcal{W} d v-\int_{\partial \kappa_{t}} \tilde{\mathbf{t}} \cdot \tilde{\chi} d a
$$

under dead-load conditions, say, where $\tilde{\boldsymbol{\chi}}(\boldsymbol{X})$ is the deformation and $\partial \kappa_{t}$ is the part of the boundary where traction is assigned. The result should be of optimal accuracy by the standard of the three-dimensional theory, and well-posed in the sense of furnishing a meaningful minimization problem in its own right.

Attention is confined to three-dimensional deformations that satisfy the strong-ellipticity condition

$$
\mathbf{a} \otimes \mathbf{b} \cdot \mathcal{M}(\tilde{\mathbf{F}})[\mathbf{a} \otimes \mathbf{b}]>0 \quad \text { for all } \mathbf{a} \otimes \mathbf{b} \neq \mathbf{0},
$$

where

$$
\mathcal{M}(\tilde{\mathbf{F}})=\mathcal{W}_{\tilde{\mathbf{F}} \tilde{\mathbf{F}}}
$$

is the tensor of elastic moduli. It is well known that this condition is necessary for minimizers of the three-dimensional energy. Later, we shall make use of the strain-dependent elastic moduli $\mathcal{C}(\tilde{\mathbf{E}})$, where

$$
\tilde{\mathbf{E}}=\frac{1}{2}\left(\tilde{\mathbf{F}}^{t} \tilde{\mathbf{F}}-\mathbf{I}\right)
$$

is the strain in which $\mathbf{I}$ is the identity for 3-space, and

$$
\mathcal{C}(\tilde{\mathbf{E}})=\mathcal{U}_{\tilde{\mathbf{E}} \tilde{\mathbf{E}}}
$$

in which

$$
\mathcal{U}(\tilde{\mathbf{E}})=\mathcal{W}(\tilde{\mathbf{F}})
$$

is the associated strain-energy function. An application of the chain rule furnishes the useful connection

$$
\mathcal{M}(\tilde{\mathbf{F}})[\mathbf{A}]=\mathbf{A} \tilde{\mathbf{S}}+\frac{1}{2} \tilde{\mathbf{F}} \mathcal{C}(\tilde{\mathbf{E}})\left[\mathbf{A}^{t} \tilde{\mathbf{F}}+\tilde{\mathbf{F}}^{t} \mathbf{A}\right]
$$

for any tensor $\mathbf{A}$, where

$$
\tilde{\mathbf{S}}=\mathcal{U}_{\tilde{\mathbf{E}}}
$$

is the symmetric second Piola-Kirchhoff stress, given in terms of the Piola stress by

$$
\tilde{\mathbf{P}}=\tilde{\mathbf{F}} \tilde{\mathbf{S}} .
$$


We assume $\mathcal{U}(\cdot)$ to be convex in a neighborhood of the origin in strain space, with the origin furnishing an isolated local minimum. Thus $\tilde{\mathbf{S}}$ vanishes at zero strain, and $\mathcal{C}(\mathbf{0})$ is positive definite on the linear space of symmetric tensors. Then,

$$
\tilde{\mathbf{S}}=\mathcal{C}(\mathbf{0})[\tilde{\mathbf{E}}]+o(|\tilde{\mathbf{E}}|) .
$$

It follows from (14), (15) and the minor symmetries of $\mathcal{C}$ that

$$
\mathcal{M}(\mathbf{I})[\mathbf{A}]=\mathcal{C}(\mathbf{0})[\mathbf{A}]
$$

and hence that our hypotheses yields strong ellipticity at zero strain, a result that is well known in linear elasticity theory. Accordingly, these hypotheses are compatible with (7).

Later, we have occasion to use the connection

$$
\mathcal{M}(\tilde{\mathbf{R}})[\mathbf{A}]=\tilde{\mathbf{R}} \mathcal{C}(\mathbf{0})\left[\tilde{\mathbf{R}}^{t} \mathbf{A}\right]
$$

where $\tilde{\mathbf{R}}$ is a rotation.

\section{Geometric and Kinematic Formulae}

We record a number of formulae pertaining to the geometry of the shell and to the kinematics of the material in a three-dimensional neighborhood of the midsurface. These either coincide with their counterparts in the linear theory or are easily obtained from them by substituting the deformation gradient in place of the displacement gradient of the linear theory. For this reason we present these matters in outline here, citing results from [4] as needed.

We seek a model for the shell involving as independent variables the coordinates $\theta^{\alpha}$ that parametrize a curved base surface $\Omega$. To this end we use the standard normal-coordinate parametrization of three dimensional space in the vicinity of the base surface [1-4]. Thus,

$$
\mathbf{X}\left(\theta^{\alpha}, \varsigma\right)=\mathbf{x}\left(\theta^{\alpha}\right)+\varsigma \mathbf{N}\left(\theta^{\alpha}\right)
$$

where $\mathbf{x}\left(\theta^{\alpha}\right)$ is the parametrization of $\Omega$ with unit-normal field $\mathbf{N}\left(\theta^{\alpha}\right)$ and $\varsigma$ is the coordinate in the direction perpendicular to $\Omega$, the latter corresponding to $\varsigma=0$. The lateral surfaces of the thin three-dimensional body are assumed to correspond to constant values of $\varsigma$. These are separated by the distance $h$, the thickness of the shell. For simplicity we assume the thickness to be uniform. Further, $h$ is assumed to be small against any other length scale at hand, such as a spanwise dimension or a local radius of curvature, and, following standard practice, we identify the base surface with the midsurface.

The orientation of $\Omega$ is induced by the assumed right-handedness of the coordinate system $\left(\theta^{\alpha}, \varsigma\right)$; thus, $\mathbf{A}_{1} \times \mathbf{A}_{2} \cdot \mathbf{N}>0$, where $\mathbf{A}_{\alpha}=\mathbf{x}_{, \alpha} \equiv \partial \mathbf{x} / \partial \theta^{\alpha}$ span the tangent plane $T_{\Omega(p)}$ to $\Omega$ at the point $p$ with coordinates $\theta^{\alpha}$. The curvature $\mathbf{B}$ of the base surface is the symmetric linear map from $T_{\Omega(p)}$ to itself defined by the Weingarten equation

$$
d \mathbf{N}=-\mathbf{B} d \mathbf{x}
$$

where $d \mathbf{x}=\mathbf{A}_{\alpha} d \theta^{\alpha}$ and $d \mathbf{N}=\mathbf{N}_{, \alpha} d \theta^{\alpha}$. Accordingly,

$$
d \mathbf{X}=d \mathbf{x}+\varsigma d \mathbf{N}+\mathbf{N} d \varsigma=\mathbf{G}(d \mathbf{x}+\mathbf{N} d \varsigma)
$$


where

$$
\mathbf{G}=\boldsymbol{\mu}+\mathbf{N} \otimes \mathbf{N}, \quad \boldsymbol{\mu}=\mathbf{1}-\varsigma \mathbf{B},
$$

and

$$
\mathbf{1}=\mathbf{I}-\mathbf{N} \otimes \mathbf{N}=\mathbf{A}_{\alpha} \otimes \mathbf{A}^{\alpha}
$$

is the projection onto - and the identity transformation for- $-T_{\Omega(p)}$, on which $\left\{\mathbf{A}^{\alpha}\right\}$ is dual to $\left\{\mathbf{A}_{\alpha}\right\}$.

In Sect. 3 we require the volume measure induced by the coordinates. This is [1-4] $d v=\mu d \varsigma d a$, where $d a=\mathbf{N} \cdot d \mathbf{x}_{1} \times d \mathbf{x}_{2}$ is the area measure on $\Omega$, and

$$
\mu=1-2 H \varsigma+\varsigma^{2} K
$$

is the (two-dimensional) determinant of $\boldsymbol{\mu}$ in which

$$
H=\frac{1}{2} \operatorname{tr} \mathbf{B} \quad \text { and } \quad K=\operatorname{det} \mathbf{B}
$$

are the mean and Gaussian curvatures of $\Omega$, respectively. This may be written $\mu=(1-$ $\left.\varsigma \kappa_{1}\right)\left(1-\varsigma \kappa_{2}\right)$, where $\kappa_{\alpha}$ - the principal curvatures - are the eigenvalues of $\mathbf{B}$. The transformation from $\left(\theta^{\alpha}, \varsigma\right)$ to $\mathbf{X}$ is one-to-one and orientation preserving if and only if $\mu>0$. Following common practice we refer to the region of space in which this condition holds as shell space. This is the region containing the base surface in which $|\varsigma|<\min \left\{r_{1}, r_{2}\right\}$, where $r_{\alpha}=\left|\kappa_{\alpha}\right|^{-1}$ are the principal radii of curvature. Equation (20) yields $d \varsigma(\mathbf{x})=\mathbf{N} \cdot d \mathbf{X}$ and hence $\mathbf{N}=D \varsigma$, implying that the normal to the base surface is also normal to the lateral surfaces of the shell. We make use of this fact in Sect. 4.

Let $C^{*}$ be the line orthogonal to $\Omega$ and intersecting $\kappa$ at a point with surface coordinates $\theta^{\alpha}$. Let $\partial \kappa_{C}=\partial \Omega \times C$, where $C$ is the collection of such lines, be the ruled generating surface of the thin shell-like region $\kappa$ obtained by translating the points of $\partial \Omega$ along their associated lines $C^{*}$. Let $s$ measure arclength on the curve $\partial \Omega$ with unit tangent $\tau$ and rightward unit normal $\boldsymbol{v}=\boldsymbol{\tau} \times \mathbf{N}$. The oriented differential surface measure induced by the $(s, \varsigma)$-parametrization of $\partial \kappa_{C}$, required in Sect. 4, is [4] $\mathbf{G}^{*} \boldsymbol{v} d s d \varsigma$, where $\mathbf{G}^{*}$ is the cofactor of $\mathbf{G}$. From this it is possible to develop a formula for the oriented area measure on the ruled generators of the shell-like body which, in turn, facilitates the development of an expression for the potential energy of an edge-loaded shell. We do not do so explicitly here, but instead quote the relevant results from [4] as needed in the sequel.

The model to be developed requires expressions for the three-dimensional deformation gradient and its through-thickness derivatives. Let $\tilde{\chi}(\mathbf{X})$ be the three-dimensional deformation with gradient $\tilde{\mathbf{F}}(\mathbf{X})=D \tilde{\chi}$. We define

$$
\hat{\chi}\left(\theta^{\alpha}, \varsigma\right)=\tilde{\chi}\left(\mathbf{x}\left(\theta^{\alpha}\right)+\varsigma \mathbf{N}\left(\theta^{\alpha}\right)\right) \quad \text { and } \quad \hat{\mathbf{F}}\left(\theta^{\alpha}, \varsigma\right)=\tilde{\mathbf{F}}\left(\mathbf{x}\left(\theta^{\alpha}\right)+\varsigma \mathbf{N}\left(\theta^{\alpha}\right)\right) \text {. }
$$

Then,

$$
\hat{\mathbf{F}}(\boldsymbol{\mu} d \mathbf{x}+\mathbf{N} d \varsigma)=d \hat{\chi}=\hat{\chi}_{, \alpha} d \theta^{\alpha}+\hat{\chi}^{\prime} d \varsigma=(\nabla \hat{\chi}) d \mathbf{x}+\hat{\chi}^{\prime} d \varsigma,
$$

where $d \theta^{\alpha}=\mathbf{A}^{\alpha} \cdot d \mathbf{x}, \hat{\chi}_{, \alpha}=\partial \hat{\chi} / \partial \theta^{\alpha}, \hat{\chi}^{\prime}=\partial \hat{\chi} / \partial \varsigma$ and

$$
\nabla \hat{\chi}=\hat{\chi}_{, \alpha} \otimes \mathbf{A}^{\alpha}
$$

is the surface deformation gradient. Thus,

$$
\hat{\mathbf{F}} \mathbf{1} \boldsymbol{\mu}=\nabla \hat{\chi} \quad \text { and } \quad \hat{\mathbf{F}} \mathbf{N}=\hat{\chi}^{\prime},
$$


in which the orthogonal decomposition $\hat{\mathbf{F}}=\hat{\mathbf{F}} \mathbf{1}+\hat{\mathbf{F}} \mathbf{N} \otimes \mathbf{N}$ has been used. Assuming the configuration $\kappa$ to be contained in shell space, we then have

$$
\hat{\mathbf{F}}=(\nabla \hat{\chi}) \boldsymbol{\mu}^{-1}+\hat{\chi}^{\prime} \otimes \mathbf{N}
$$

The mid-surface value of $\hat{\mathbf{F}}$, and those of its through-thickness derivatives $\hat{\mathbf{F}}^{\prime}$ and $\hat{\mathbf{F}}^{\prime \prime}$, are needed in Sect. 4. These, and other variables defined on the midsurface, are identified by the absence of superposed carets. Following the procedure discussed in [4], they are found to be

$$
\mathbf{F}=\nabla \mathbf{r}+\mathbf{d} \otimes \mathbf{N}, \quad \mathbf{F}^{\prime}=\nabla \mathbf{d}+(\nabla \mathbf{r}) \mathbf{B}+\mathbf{g} \otimes \mathbf{N}
$$

and

$$
\mathbf{F}^{\prime \prime}=\nabla \mathbf{g}+2(\nabla \mathbf{d}) \mathbf{B}+2(\nabla \mathbf{r}) \mathbf{B}^{2}+\mathbf{h} \otimes \mathbf{N}
$$

where

$$
\mathbf{r}=\chi, \quad \mathbf{d}=\chi^{\prime}, \quad \mathbf{g}=\chi^{\prime \prime} \quad \text { and } \quad \mathbf{h}=\chi^{\prime \prime \prime}
$$

are mutually independent functions of $\theta^{\alpha}$ and the operation $\nabla(\cdot)$ is defined by (27). The first of these is the mid-surface deformation field and the latter three are the directors. Together they furnish the coefficient vectors in the expansion

$$
\hat{\chi}=\mathbf{r}+\varsigma \mathbf{d}+\frac{1}{2} \varsigma^{2} \mathbf{g}+\frac{1}{6} \varsigma^{3} \mathbf{h}+\cdots
$$

of the three-dimensional deformation, where $\mathbf{r}\left(\theta^{\alpha}\right)=\hat{\chi}\left(\mathbf{x}\left(\theta^{\alpha}\right)\right)$ is the position of a material point on the deformed image $\omega$ of the midsurface $\Omega$; its gradient $\nabla \mathbf{r}$ maps $T_{\Omega(p)}$ to the tangent plane $T_{\omega(p)}$ to $\omega$ at the material point $p$.

We note that the regularity of the three-dimensional deformation required by the expansion (33) is not implied by Ball's existence theory for equilibria [5]. Nevertheless any piecewise $C^{2}$ equilibrium deformation, possessing a potential jump in its normal derivative across a smooth surface in $\kappa$, is in fact $C^{2}$ in the presence of strong ellipticity. It is straightforward to show that it is then $C^{n}$ for arbitrary $n$. Further, in [6] strong ellipticity is used with degree-theoretic arguments to obtain partial existence results for classically smooth (i.e., $C^{2}$ ) equilibria. Given our adoption of strong ellipticity it is thus appropriate to assume that equilibria are sufficiently smooth to justify (33), and to confine attention to deformations that satisfy it a priori. It is well known that such deformations are not rich enough to yield the optimal leading-order stretching (membrane) energy [7]. However, the latter may be identified with the relaxation [8] of the stretching energy found here, as explained in [9].

\section{Expansion of the Three-dimensional Energy}

The strain energy of the shell is

$$
\int_{\kappa} \mathcal{W}(\hat{\mathbf{F}}) d v=\int_{\Omega} W d a,
$$

where

$$
W=\int_{C} \mu \mathcal{W}(\hat{\mathbf{F}}) d \varsigma
$$


with $C=[-h / 2, h / 2]$, is the areal strain-energy density on $\Omega$. For a given deformation this may be regarded as a function of $h$. Our smoothness assumptions are then consistent with the Taylor expansion [4]

$$
W=h\left(1+\frac{1}{12} h^{2} K\right) \mathcal{W}+\frac{1}{24} h^{3}\left(\mathcal{W}^{\prime \prime}-4 H \mathcal{W}^{\prime}\right)+o\left(h^{3}\right)
$$

obtained with the aid of Leibniz' rule, where, for uniform materials,

$$
\mathcal{W}=\mathcal{W}(\mathbf{F}), \quad \mathcal{W}^{\prime}=\mathbf{P} \cdot \mathbf{F}^{\prime}, \quad \text { and } \quad \mathcal{W}^{\prime \prime}=\mathbf{P}^{\prime} \cdot \mathbf{F}^{\prime}+\mathbf{P} \cdot \mathbf{F}^{\prime \prime}
$$

with

$$
\mathbf{P}^{\prime}=\mathcal{M}(\mathbf{F})\left[\mathbf{F}^{\prime}\right]
$$

The development is eased considerably by using the decompositions (cf. (22))

$$
\mathbf{P}=\mathbf{P} 1+\mathbf{P N} \otimes \mathbf{N} \quad \text { and } \quad \mathbf{P}^{\prime}=\mathbf{P}^{\prime} \mathbf{1}+\mathbf{P}^{\prime} \mathbf{N} \otimes \mathbf{N}
$$

in (37), leading to

$$
\mathcal{W}^{\prime}=\mathbf{P 1} \cdot[\nabla \mathbf{d}+(\nabla \mathbf{r}) \mathbf{B}]+\mathbf{P N} \cdot \mathbf{g}
$$

and

$$
\mathcal{W}^{\prime \prime}=\mathbf{P}^{\prime} \mathbf{1} \cdot[\nabla \mathbf{d}+(\nabla \mathbf{r}) \mathbf{B}]+\mathbf{P}^{\prime} \mathbf{N} \cdot \mathbf{g}+\mathbf{P} \mathbf{1} \cdot\left[\nabla \mathbf{g}+2(\nabla \mathbf{d}) \mathbf{B}+2(\nabla \mathbf{r}) \mathbf{B}^{2}\right]+\mathbf{P N} \cdot \mathbf{h} .
$$

Assuming the lateral surfaces to be traction free, the load potential in (6) is found, precisely as in [4], to be

$$
\int_{\partial \kappa_{t}} \tilde{\mathbf{t}} \cdot \tilde{\chi} d a=\int_{\partial \Omega_{t}}\left(\mathbf{p}_{r} \cdot \mathbf{r}+\mathbf{p}_{d} \cdot \mathbf{d}+\mathbf{p}_{g} \cdot \mathbf{g}\right) d s+o\left(h^{3}\right),
$$

where

$$
\begin{aligned}
& \mathbf{p}_{r}=h\left(1+\frac{1}{24} h^{2} \tau^{2}\right) \mathbf{t}+\frac{1}{24} h^{3}\left(\mathbf{t}^{\prime \prime}-2 \kappa_{\tau} \mathbf{t}^{\prime}\right), \quad \mathbf{p}_{d}=\frac{1}{12} h^{3}\left(\mathbf{t}^{\prime}-\kappa_{\tau} \mathbf{t}\right), \\
& \mathbf{p}_{g}=\frac{1}{24} h^{3} \mathbf{t},
\end{aligned}
$$

and

$$
\begin{aligned}
& \mathbf{t}=\mathbf{P} \mathbf{1} v, \quad \mathbf{t}^{\prime}-\kappa_{\tau} \mathbf{t}=\mathbf{P}^{\prime} \mathbf{1} v+\tau \mathbf{P} \mathbf{1} \tau-\kappa_{\tau} \mathbf{P} \mathbf{1} v, \\
& \mathbf{t}^{\prime \prime}-2 \kappa_{\tau} \mathbf{t}^{\prime}=\mathbf{P}^{\prime \prime} \mathbf{1} v+2\left(\tau \mathbf{P}^{\prime} \mathbf{1} \tau-\kappa_{\tau} \mathbf{P}^{\prime} \mathbf{1} v\right)-\tau^{2} \mathbf{P} \mathbf{1} v
\end{aligned}
$$

in which $\kappa_{\tau}$ is the normal curvature of $\partial \Omega$ and $\tau$ is the twist. The latter formulas follow by expanding the oriented area measure, alluded to previously, on the ruled generators of the shell [4]. 


\section{The Optimum Order- $h^{3}$ Energy}

We seek an order- $h^{3}$ truncation of the potential energy that is as accurate as possible by the standard of three-dimensional elasticity theory. To this end we impose restrictions on the director fields $\mathbf{d}, \mathbf{g}$, etc. as required by that theory. Thus we use partial information about three-dimensional equilibria to generate the optimal order- $h^{3}$ potential energy functional for the midsurface deformation. In other words, although at this stage $\mathbf{r}\left(\theta^{\alpha}\right)$ is unrestricted, the expression for $E$, to be derived, is not valid for arbitrary kinematically possible threedimensional states. Instead it is defined on a manifold of configurations parametrized by $\mathbf{r}\left(\theta^{\alpha}\right)$, defined by equilibrium constraints on the directors.

For example, the exact expressions $\tilde{\mathbf{t}}^{+}=\tilde{\mathbf{P}}^{+} \mathbf{N}$ and $\tilde{\mathbf{t}}^{+}=-\tilde{\mathbf{P}}^{-} \mathbf{N}$ for the tractions at the lateral surfaces with exterior unit normals $\pm \mathbf{N}$ (cf. Sect. 2), together with Taylor expansions of $\tilde{\mathbf{P}}^{ \pm}$, furnish

$$
\tilde{\mathbf{t}}^{+}+\tilde{\mathbf{t}}^{-}=h \mathbf{P}^{\prime} \mathbf{N}+O\left(h^{3}\right) \quad \text { and } \quad \tilde{\mathbf{t}}^{+}-\tilde{\mathbf{t}}^{-}=2 \mathbf{P N}+O\left(h^{2}\right)
$$

Accordingly, for traction-free lateral surfaces,

$$
\mathbf{P N}, \mathbf{P}^{\prime} \mathbf{N}=O\left(h^{2}\right),
$$

and with these in hand it follows that the terms in (36) involving $\mathbf{P N}$ and $\mathbf{P}^{\prime} \mathbf{N}$ in the coefficient of $h^{3}$ may be dropped. Thus, (36) remains valid with the simplifications

$$
\mathcal{W}^{\prime}=\overline{\mathbf{P}} \mathbf{1} \cdot[\nabla \overline{\mathbf{d}}+(\nabla \mathbf{r}) \mathbf{B}]
$$

and

$$
\mathcal{W}^{\prime \prime}=\overline{\mathbf{P}}^{\prime} \mathbf{1} \cdot[\nabla \overline{\mathbf{d}}+(\nabla \mathbf{r}) \mathbf{B}]+\overline{\mathbf{P}} \mathbf{1} \cdot\left[\nabla \overline{\mathbf{g}}+2(\nabla \overline{\mathbf{d}}) \mathbf{B}+2(\nabla \mathbf{r}) \mathbf{B}^{2}\right]
$$

in which $\overline{\mathbf{d}}$ and $\overline{\mathbf{g}}$ satisfy the constraints $\mathbf{P N}=\mathbf{0}$ and $\mathbf{P}^{\prime} \mathbf{N}=\mathbf{0}$, respectively, i.e.,

$$
\begin{aligned}
& \{\tilde{\mathbf{P}}(\nabla \mathbf{r}+\overline{\mathbf{d}} \otimes \mathbf{N})\} \mathbf{N}=\mathbf{0} \text { and } \\
& \{\mathcal{A}(\nabla \mathbf{r}+\overline{\mathbf{d}} \otimes \mathbf{N})\} \overline{\mathbf{g}}=-\{\mathcal{M}(\nabla \mathbf{r}+\overline{\mathbf{d}} \otimes \mathbf{N})[\nabla \overline{\mathbf{d}}+(\nabla \mathbf{r}) \mathbf{B}]\} \mathbf{N},
\end{aligned}
$$

and where $\mathcal{A}$ is the acoustic tensor defined by

$$
\mathcal{A}(\tilde{\mathbf{F}}) \mathbf{v}=\{\mathcal{M}(\tilde{\mathbf{F}})[\mathbf{v} \otimes \mathbf{N}]\} \mathbf{N}
$$

This of course is positive definite by virtue of strong ellipticity, and $(49)_{2}$ then furnishes $\overline{\mathbf{g}}$ uniquely. That (49) $)_{1}$ possesses a unique solution also follows from strong ellipticity; this is demonstrated in [10,11]. The notation $\overline{\mathbf{P}}$ and $\overline{\mathbf{P}}^{\prime}$ in (48) refers to the midsurface values of the stress and its $\varsigma$-derivative in which $\mathbf{d}=\overline{\mathbf{d}}$ and $\mathbf{g}=\overline{\mathbf{g}}$ are imposed. We observe, however, that our argument does not yield $\mathbf{d}=\overline{\mathbf{d}}$ in the coefficient of $h$ in the expression (36) for the strain-energy density. Indeed, if this is imposed then in principle an error is incurred which is of the same order as the order- $h^{3}$ term exhibited explicitly.

Remark It is shown in [12] that the solution $\overline{\mathbf{d}}$ to $(49)_{1}$ minimizes the midsurface strain energy $\mathcal{W}(\nabla \mathbf{r}+\mathbf{d} \otimes \mathbf{N})$ with respect to $\mathbf{d}$ and that the solution $\overline{\mathbf{g}}$ to $(49)_{2}$ minimizes the quadratic form $(\nabla \overline{\mathbf{d}}+(\nabla \mathbf{r}) \mathbf{B}+\mathbf{g} \otimes \mathbf{N}) \cdot \mathcal{M}(\mathbf{F})[\nabla \overline{\mathbf{d}}+(\nabla \mathbf{r}) \mathbf{B}+\mathbf{g} \otimes \mathbf{N}]$ with respect to $\mathbf{g}$. 
These claims also follow from strong ellipticity. The first result justifies the imposition of $\mathbf{d}=\overline{\mathbf{d}}$ in all terms of the energy, yielding (36), with (47) and (48), in which

$$
\mathcal{W}=\mathcal{W}(\nabla \mathbf{r}+\overline{\mathbf{d}} \otimes \mathbf{N}) \quad \text { and } \quad \overline{\mathbf{P}}^{\prime} \mathbf{1} \cdot[\nabla \overline{\mathbf{d}}+(\nabla \mathbf{r}) \mathbf{B}]=\mathbf{K} \cdot \mathcal{M}(\nabla \mathbf{r}+\overline{\mathbf{d}} \otimes \mathbf{N})[\mathbf{K}]
$$

where

$$
\mathbf{K}=\nabla \overline{\mathbf{d}}+(\nabla \mathbf{r}) \mathbf{B}+\overline{\mathbf{g}} \otimes \mathbf{N}
$$

The term P1 $\nabla \mathbf{g}$ appearing in (48) may be reduced with the aid of the equilibrium (4). We write the latter in the form $\left(\tilde{\mathbf{P}}_{, i}\right) \mathbf{G}^{i}=\mathbf{0}$, where $\mathbf{G}^{i}=D \theta^{i}(\mathbf{X})$ and $\left\{\theta^{i}\right\}=\left\{\theta^{\alpha}, \varsigma\right\}$. This is well defined in shell space and hence on the midsurface, where it reduces to $\left(\mathbf{P}_{, \alpha}\right) \mathbf{A}^{\alpha}+\mathbf{P}^{\prime} \mathbf{N}=\mathbf{0}$. With some effort this may be recast as [4]

$$
\operatorname{div}(\mathbf{P 1})+\mathbf{P}^{\prime} \mathbf{N}-2 H \mathbf{P N}=\mathbf{0},
$$

where

$$
\operatorname{div}(\mathbf{P 1})=\mathbf{P}_{\mid \alpha}^{\alpha}=A^{-1 / 2}\left(A^{1 / 2} \mathbf{P}^{\alpha}\right)_{, \alpha},
$$

in which $\mathbf{P}^{\alpha}=\mathbf{P A}^{\alpha}, A=\operatorname{det}\left(A_{\alpha \beta}\right)$ and $A_{\alpha \beta}=\mathbf{A}_{\alpha} \cdot \mathbf{A}_{\beta}$ is the (positive definite) metric on $\Omega$ induced by the parametrization $\mathbf{x}\left(\theta^{\alpha}\right)$. Equation (53) is amenable to the application of Stokes' theorem, which furnishes [4]

$$
\int_{\Omega} \mathbf{P} \mathbf{1} \cdot \nabla \mathbf{g} d a=\int_{\partial \Omega} \mathbf{P} \mathbf{1} \mathbf{v} \cdot \mathbf{g} d s+\int_{\Omega} \mathbf{g} \cdot\left(\mathbf{P}^{\prime} \mathbf{N}-2 H \mathbf{P N}\right) d a .
$$

Using (46), (47) and (48) we thus derive

$$
\int_{\kappa} \mathcal{W} d v=\int_{\Omega} W d a+\frac{1}{24} h^{3} \int_{\partial \Omega} \overline{\mathbf{P}} \mathbf{1} v \cdot \overline{\mathbf{g}} d s
$$

where $W$ is now given by

$$
W=\left(1+\frac{1}{12} h^{2} K\right) W_{1}+W_{2}+W_{3}+o\left(h^{3}\right),
$$

with

$$
\begin{aligned}
& W_{1}=h \mathcal{W}(\nabla \mathbf{r}+\overline{\mathbf{d}} \otimes \mathbf{N}), \quad W_{2}=\frac{1}{24} h^{3} \mathbf{K} \cdot \mathcal{M}(\nabla \mathbf{r}+\overline{\mathbf{d}} \otimes \mathbf{N})[\mathbf{K}] \quad \text { and } \\
& W_{3}=\frac{1}{12} h^{3} \overline{\mathbf{P}} \mathbf{1} \cdot\left\{\left[(\nabla \overline{\mathbf{d}}) \mathbf{B}+(\nabla \mathbf{r}) \mathbf{B}^{2}\right]-2 H[\nabla \overline{\mathbf{d}}+(\nabla \mathbf{r}) \mathbf{B}]\right\}
\end{aligned}
$$

The term $W_{3}$ is non-standard. Its counterpart in Koiter's work [3] was the subject of extensive analysis, the rough conclusion of which is that it may be neglected in comparison to the sum $W_{1}+W_{2}$, provided that the midsurface strain is small and the shell is sufficiently thin. We return to this issue below. Evidently $W=W_{1}+W_{2}$ if the midsurface is flat, i.e. if the shell is a plate [12]. 


\section{Reflection Symmetry}

It is usual, when deriving shell theory from the three-dimensional theory, to restrict attention to materials that exhibit reflection symmetry of the material properties with respect to the midsurface. These are exemplified by the case of isotropy, discussed by Koiter, which we develop in detail. In the classical linear theory of flat plates, this generates a model in which the in-plane and bending displacements decouple at leading order. It also yields important simplifications in the case of shells, the absence of such decoupling notwithstanding. Thus we consider strain-energy functions that satisfy $\mathcal{U}(\mathbf{E})=\mathcal{U}\left(\mathbf{Q}^{t} \mathbf{E} \mathbf{Q}\right)$ with $\mathbf{Q}=\mathbf{I}-2 \mathbf{N} \otimes \mathbf{N}$, which in turn implies that $\mathcal{U}^{\prime}\left(E_{i j}\right)=\mathcal{U}\left(E_{k l} \mathbf{G}^{k} \otimes \mathbf{G}^{l}\right)$, where $\mathbf{G}^{\alpha}=\mathbf{A}^{\alpha}$ and $\mathbf{G}^{3}=\mathbf{N}$, is an even function of the transverse shear strain $E_{\alpha 3}\left(=E_{3 \alpha}\right)$.

Let $\Gamma\left(E_{\alpha 3}\right)$ be the function obtained by fixing all components of $\mathbf{E}$ except $E_{\alpha 3}$ in the midsurface strain-energy function. Then,

$$
\partial \Gamma / \partial E_{\alpha 3}=\mathbf{A}^{\alpha} \cdot\left(\mathcal{U}_{\mathbf{E}}\right) \mathbf{N}
$$

which vanish by (13) and (49) $)_{1}$, the latter being equivalent to

$$
\mathbf{S N}=\mathbf{0} \text {. }
$$

In materials that exhibit reflection symmetry these restrictions are satisfied at $E_{\alpha 3}=0$ because the strain energy is then an even function of the transverse shears [10]. The corresponding strain is

$$
\mathbf{E}=\boldsymbol{\epsilon}+E_{33} \mathbf{N} \otimes \mathbf{N}, \quad \text { where } \boldsymbol{\epsilon}=E_{\alpha \beta} \mathbf{A}^{\alpha} \otimes \mathbf{A}^{\beta}, \quad E_{33}=\frac{1}{2}\left(\varphi^{2}-1\right)
$$

and $\varphi$ is the transverse stretch. Comparing with the midsurface strain obtained from (30) 1 , we conclude that

$$
\mathbf{d}=\varphi \mathbf{n},
$$

where $\mathbf{n}$ is the unit normal to the deformed surface defined by $\left|\mathbf{F}^{*} \mathbf{N}\right| \mathbf{n}=\mathbf{F}^{*} \mathbf{N}$, where $\mathbf{F}^{*}$ is the cofactor of $\mathbf{F}$, and where $\varphi(>0)$ is obtained in terms of $\boldsymbol{\epsilon}$ by solving

$$
\mathbf{N} \cdot \mathbf{S N}=0 .
$$

That (62) furnishes a solution to (60) in the presence of reflection symmetry was proved in $[2,10]$. The energetic optimality of the solution, granted strong ellipticity, was proved in [12, p. 288]. Therefore reflection symmetry and strong ellipticity, combined with (60), yield deformations in which the transverse shear strain necessarily vanishes and $\mathbf{S}$ is a symmetric 2-tensor that maps the tangent plane $T_{\Omega(p)}$ to itself.

For the truncated energy exhibited explicitly in (56) to furnish a meaningful minimization problem in its own right, it is necessary that a minimizing configuration satisfy the operative Legendre-Hadamard condition pointwise in $\Omega$. Because the integrand $W$ involves $\nabla \mathbf{r}$ and $\nabla \nabla \mathbf{r}$ in which dependence on the latter is quadratic, this amounts to the requirement that the homogeneous quadratic term $W_{2}$ be positive definite when $\nabla \nabla \mathbf{r}$ is replaced by $\mathbf{y} \otimes \mathbf{z} \otimes \mathbf{z}$, for any three-vector $\mathbf{y}$ and any two-vector $\mathbf{z} \in T_{\Omega(p)}$ [13]. Choosing $\mathbf{y}$ such that $\mathbf{N} \cdot \mathbf{y}=0$ reduces this requirement, in the case of reflection symmetry, to the restriction

$$
\nabla \varphi \cdot \mathbf{S}(\nabla \varphi) \geq 0
$$


in which $\nabla \varphi$ can be assigned arbitrary values by choice of $\mathbf{z}$ and the projection $\mathbf{1 y}$. The proof, which makes use of (12), is given in [11], where a truncated strain energy equivalent to our $W_{1}+W_{2}$ is derived for plates. The requirement thus limits the present model to states of midsurface stress that are pointwise positive definite. This of course does not portend a deficiency of the theory, because minimizers of the actual three-dimensional energy need not minimize finite-order truncations of the energy. Nevertheless the issue does pose an obstacle to analysis based on the truncated energy. In [11] this is addressed by adding an ad hoc strain-gradient term arranged to ensure that the Legendre-Hadamard condition is automatically satisfied. Here, we pursue an alternative that culminates in Koiter's expression for the energy.

\section{Koiter's Energy as the Leading-Order Model}

\subsection{Restriction to Small Midsurface Strain}

The restriction (64) to positive-definite stress $\mathbf{S}$ is associated with the coefficient of $h^{3}$ in the strain-energy function. To remove it, and thus to restore the potential well-posedness of the truncated shell energy (56) when the pointwise restriction (64) is violated, we assume that $\mathbf{S}$ vanishes with shell thickness, i.e., that

$$
|\mathbf{S}|=o(1)
$$

after suitable non-dimensionalization. This assumption must be verified a posteriori. Indeed, the connection between shell thickness and the magnitude of the midsurface stress, and consequently that of the midsurface strain, lies at the heart of the ubiquitous claim made in the technical literature, either tacitly or explicitly $[1,2]$, to the effect that thin shells typically undergo finite deformations with small midsurface strains. Here we interpret this view to mean that the stress scales with shell thickness; our assumption (65) is the most conservative restriction of this kind.

This reasoning amounts to a conjecture that states which involve bending and which are such as to violate the Legendre-Hadamard condition (64) are accommodated, in stable configurations, by a reduction in the magnitude of stress, and associated midsurface strain, precisely so that equilibria then furnish minima of the dominant contribution to the overall energy. Said differently, to the extent that the order- $h^{3}$ truncation of the energy furnishes a meaningful approximation to the energy for shells of small thickness, it is necessary that the stress in a stable configuration scale as indicated whenever it fails to be pointwise positive definite. From this point of view the restriction to small midsurface strain, discussed on an anecdotal basis in the literature, may be motivated on energetic grounds, at least for stable equilibria.

We thus have $h^{3} \mathbf{S}=o\left(h^{3}\right)$, and from $\mathbf{P 1}=(\nabla \mathbf{r}) \mathbf{S}$ we conclude that $W_{3}=o\left(h^{3}\right)$. This implies that the involved estimates of $W_{3}$ underlying Koiter's approach [3] are not required. Further, the constitutive hypotheses discussed in Sect. 1 imply that the midsurface value of the strain is sufficiently small-of order $o(1)$ - that it may be neglected in the coefficient of $h^{3}$ without adverse effect on the accuracy of (56). Suppressing, in addition, the small term $h^{2} K$ in comparison to unity, we arrive at

$$
\int_{\kappa} \mathcal{W}(\hat{\mathbf{F}}) d v=\int_{\Omega} W d a, \quad \text { with } W=W_{1}+W_{2}+o\left(h^{3}\right)
$$


in which $W_{2}$ is now given by

$$
W_{2}=\frac{1}{24} h^{3} \mathbf{K} \cdot \mathcal{M}(\mathbf{R})[\mathbf{K}]
$$

where $\mathbf{R}$ is the rotation factor in the polar decomposition of $\overline{\mathbf{F}}=\nabla \mathbf{r}+\overline{\mathbf{d}} \otimes \mathbf{N}$; that is, the replacement of $\overline{\mathbf{F}}$ by $\mathbf{R}$ entails an error of order $o(1)$, provided that the strain-energy function is sufficiently smooth, and thus an overall error of order $o\left(h^{3}\right)$ which does not affect the order- $h^{3}$ truncation. Using (17) we then have

$$
W_{2}=\frac{1}{24} h^{3} \mathbf{R}^{t} \mathbf{K} \cdot \mathcal{C}(\mathbf{0})\left[\mathbf{R}^{t} \mathbf{K}\right],
$$

with $\mathbf{K}$ given by (52), where (cf. (62))

$$
\nabla \overline{\mathbf{d}}=\nabla \mathbf{n}+\mathbf{n} \otimes \nabla \varphi
$$

in which $\varphi=1$ has been imposed for consistency with the suppression of the strain in the coefficient of $h^{3}$. For consistency we also impose

$$
\nabla \mathbf{r}=\overline{\mathbf{F}} \mathbf{1}=\mathbf{R} \mathbf{1} \text { and } \mathbf{n}=\mathbf{R} \mathbf{N}
$$

in this coefficient. The consistent computation of $\overline{\mathbf{g}}$ entails the replacement of $(49)_{2}$ by

$$
\{\mathcal{A}(\mathbf{R})\} \overline{\mathbf{g}}=-\{\mathcal{M}(\mathbf{R})[\nabla \overline{\mathbf{d}}+(\nabla \mathbf{r}) \mathbf{B}]\} \mathbf{N},
$$

where (cf. (50))

$$
\mathcal{A}(\mathbf{R}) \mathbf{v}=\{\mathcal{M}(\mathbf{R})[\mathbf{v} \otimes \mathbf{N}]\} \mathbf{N} .
$$

Using (17), (71) may then be cast in the form

$$
\left\{\mathcal{C}(\mathbf{0})\left[\mathbf{R}^{t} \overline{\mathbf{g}} \otimes \mathbf{N}\right]\right\} \mathbf{N}=-\left\{\mathcal{C}(\mathbf{0})\left[\mathbf{R}^{t} \nabla \overline{\mathbf{d}}+\mathbf{B}\right]\right\} \mathbf{N} .
$$

The strain energy is thus given by (66) in which

$$
W=h \mathcal{W}(\nabla \mathbf{r}+\overline{\mathbf{d}} \otimes \mathbf{N})+\frac{1}{24} h^{3} \mathbf{R}^{t} \mathbf{K} \cdot \mathcal{C}(\mathbf{0})\left[\mathbf{R}^{t} \mathbf{K}\right],
$$

with K computed as indicated. Details of the calculation are given below. The load potential (42) may also be simplified; we obtain

$$
\int_{\partial \kappa_{t}} \tilde{\mathbf{t}} \cdot \tilde{\chi} d a=\int_{\partial \Omega_{t}}\left(\mathbf{p}_{r} \cdot \mathbf{r}+\mathbf{p}_{d} \cdot \overline{\mathbf{d}}\right) d s+o\left(h^{3}\right),
$$

in which

$$
\mathbf{p}_{r}=h \mathbf{t}+\frac{1}{24} h^{3}\left(\mathbf{t}^{\prime \prime}-2 \kappa_{\tau} \mathbf{t}^{\prime}\right), \quad \mathbf{p}_{d}=\frac{1}{12} h^{3} \mathbf{t}^{\prime}
$$

and the small term $\frac{1}{24} h^{2} \tau^{2}$ has been neglected in comparison to unity.

Here we have assumed $\mathbf{d}=\overline{\mathbf{d}}$ and $\mathbf{g}=\overline{\mathbf{g}}$ to obtain on the closure of $\Omega$. Regarding boundary data, we assume that $\tilde{\boldsymbol{\chi}}$ is assigned on $\left(\partial \Omega \backslash \partial \Omega_{t}\right) \times[-h / 2, h / 2]$. This implies that its midsurface value, $\mathbf{r}$, and those of its tangential through-thickness derivatives, $\mathbf{d}$ and $\mathbf{g}$, 
are also assigned there. However, the latter two fields cannot be assigned arbitrarily if the foregoing model is to apply on the closure of $\Omega$. The assigned values must agree with the continuous extensions to $\partial \Omega \backslash \partial \Omega_{t}$ of the functions $\overline{\mathbf{d}}$ and $\overline{\mathbf{g}}$, respectively. If these extensions are in conflict with the values derived from the data for the three-dimensional problem - this situation being typical - then it is necessary to use three-dimensional theory (or some refinement of the present theory) in a region adjoining the boundary; one then attempts to match its predictions to those of the present model in the interior.

\subsection{Koiter's Energy as the Leading-Order Model}

If the midsurface stress scales as

$$
\mathbf{S}=h \overline{\mathbf{S}}+h^{2} \stackrel{\circ}{\mathbf{S}}+o\left(h^{2}\right)
$$

with $\overline{\mathbf{S}}$ and $\mathbf{S}$ independent of $h$, then our constitutive hypotheses furnish the strain

$$
\mathbf{E}=h \overline{\mathbf{E}}+o(h),
$$

where $\overline{\mathbf{E}}=\mathcal{S}[\overline{\mathbf{S}}]$ and $\mathcal{S}$ is the compliance tensor (the inverse of $\mathcal{C}(\mathbf{0})$ ). Scalings of this kind are sometimes assumed a priori in asymptotic treatments (e.g., [14]). The term $W_{1}$ in the strain energy (cf. (58)) reduces to

$$
W_{1}=\frac{1}{2} h^{3} \overline{\mathbf{E}} \cdot \mathcal{C}(\mathbf{0})[\overline{\mathbf{E}}]+o\left(h^{3}\right),
$$

yielding

$$
W=h^{3} \bar{W}+o\left(h^{3}\right)
$$

where

$$
\bar{W}=\frac{1}{2} \overline{\mathbf{E}} \cdot \mathcal{C}(\mathbf{0})[\overline{\mathbf{E}}]+\frac{1}{24} \mathbf{R}^{t} \mathbf{K} \cdot \mathcal{C}(\mathbf{0})\left[\mathbf{R}^{t} \mathbf{K}\right] .
$$

We show below that this coincides with Koiter's expression for the shell strain-energy density.

In consequence of (43) and (44) we also have

$$
\mathbf{p}_{r} \cdot \mathbf{r}+\mathbf{p}_{d} \cdot \overline{\mathbf{d}}=h^{3}\left(\overline{\mathbf{p}}_{r} \cdot \mathbf{r}+\overline{\mathbf{p}}_{d} \cdot \overline{\mathbf{d}}\right)+o\left(h^{3}\right)
$$

where

$$
\overline{\mathbf{p}}_{r}=\stackrel{\circ}{\mathbf{t}}+\frac{1}{24}\left(\mathbf{t}^{\prime \prime}-2 \kappa_{\tau} \mathbf{t}^{\prime}\right), \quad \overline{\mathbf{p}}_{d}=\frac{1}{12} \mathbf{t}^{\prime} \quad \text { with } \stackrel{\circ}{\mathbf{t}}=\mathbf{R} \stackrel{\circ}{\mathbf{S} v}
$$

and we have imposed $\overline{\mathbf{S}} \boldsymbol{v}=\mathbf{0}$ on $\partial \Omega_{t}$ to suppress an order- $h^{2}$ term in the load potential. The latter assumption must also, in principle, be checked a posteriori.

Altogether, the potential energy $\mathcal{E}$ is then given by

$$
\mathcal{E} / h^{3}=\bar{E}+o\left(h^{3}\right) / h^{3}
$$

where

$$
\bar{E}=\int_{\Omega} \bar{W} d a-\int_{\partial \Omega_{t}}\left(\overline{\mathbf{p}}_{r} \cdot \mathbf{r}+\overline{\mathbf{p}}_{d} \cdot \overline{\mathbf{d}}\right) d s
$$


yielding $E=h^{3} \bar{E}$ in (5). This is the leading order potential energy of the shell under the present hypotheses. In view of (84), it would be possible to regard $\bar{E}$ as the rigorous leadingorder energy, in the sense of gamma convergence or asymptotic analysis, if the estimate (77) on the (plane) stress could be established a priori in the interior of $\Omega$. As it stands, the only rationale for (77) that we know of is that it yields an expression for the leading-order energy which furnishes a meaningful minimization problem. Examples of such states include, rather obviously, the minimizers (if any) of $\bar{E}$.

We note that the separate stretching and bending energies in (74), based on the hypothesis that the (three-dimensional) material is of the linear Kirchhoff-St. Venant type and hence that $W_{1}=\frac{1}{2} h \mathbf{E} \cdot \mathcal{C}(\mathbf{0})[\mathbf{E}]$, have been justified via asymptotic analysis of the weak forms of the equilibrium equations [15-17]. However, the a priori restriction to small strain implicit in the Kirchhoff-St. Venant model seems not to have been justified. From the present perspective the restriction to small strain may be motivated by requiring the order- $h^{3}$ truncation (56) of the energy to furnish a meaningful minimization problem, the appropriateness of the requirement then hinging, post facto, on the existence of minimizers of the truncated energy. If, in addition, the stress scales in accordance with (77), then bending and stretching effects are of comparable importance and Koiter's combined bending-stretching model emerges as the rigorous leading-order energy.

For stresses that satisfy the weaker restriction (65), the energy (74) furnishes the natural extension of Koiter's to the case of finite midsurface strain. The separate order- $h$ and order- $h^{3}$ terms therein may also be justified via asymptotic analysis in the manner of [1517] and [18, Sect. 3], and, as remarked by Koiter [19], the composite energy may then be regarded as furnishing leading-order models in different regions of the shell; the first (order $h$ ), wherever the midsurface strain is non-zero, and the second (order $h^{3}$ ), where bending effects dominate. A weakly nonlinear extension of the Kirchhoff-St. Venant model is then appropriate (see [20], Sect. 7). However, in the absence of a precise scaling in place of (65), it is necessary to retain the given constitutive function $\mathcal{W}$ in (74).

The associated Euler equations and boundary conditions are well known and will not be presented here. It is customary to express them in terms of covariant derivatives and geometric parameters on the deformed surface $[1,2]$. To connect the boundary conditions to the load potential in (85), we show that the variation of the latter may be expressed in the form

$$
\int_{\partial \Omega_{t}}\left[\overline{\mathbf{p}}_{r} \cdot \mathbf{u}+\overline{\mathbf{p}}_{d} \cdot(\overline{\mathbf{d}})^{\cdot}\right] d s=\int_{\partial \omega_{t}}(\mathbf{f} \cdot \mathbf{u}-M \overline{\boldsymbol{\tau}} \cdot \boldsymbol{\omega}) d \bar{s},
$$

where $\mathbf{u}=\dot{\mathbf{r}}$, superposed dots are used to denote variational (Gateaux) derivatives, $\mathbf{f}$ is the net force density on the deformed edge with unit tangent $\overline{\boldsymbol{\tau}}, \boldsymbol{\omega}$ is the virtual rotation of the shell midsurface defined by $\dot{\mathbf{n}}=\boldsymbol{\omega} \times \mathbf{n}$ and $M$ is the density of bending couple along the edge.

To this end we observe that to within negligible errors of order $h$, arclength on $\partial \Omega$ coincides with that on $\partial \omega$, and $\overline{\mathbf{d}}=\mathbf{n}$ (see (62)), whereas [21]

$$
\dot{\mathbf{n}}=(\overline{\boldsymbol{\tau}} \cdot \boldsymbol{\omega}) \overline{\boldsymbol{v}}-\left(\mathbf{n} \cdot \mathbf{u}_{, \bar{s}}\right) \overline{\boldsymbol{\tau}},
$$

where $\overline{\boldsymbol{v}}=\overline{\boldsymbol{\tau}} \times \mathbf{n}$ and $(\cdot)_{, \bar{s}}=d(\cdot) / d \bar{s}$. Thus, to consistent order,

$$
\overline{\mathbf{p}}_{r} \cdot \mathbf{u}+\overline{\mathbf{p}}_{d} \cdot(\overline{\mathbf{d}})^{\cdot}=\left\{\overline{\mathbf{p}}_{r}-\left[\left(\overline{\boldsymbol{\tau}} \cdot \overline{\mathbf{p}}_{d}\right) \mathbf{n}\right]_{, \bar{s}}\right\} \cdot \mathbf{u}+\left(\overline{\boldsymbol{v}} \cdot \overline{\mathbf{p}}_{d}\right) \overline{\boldsymbol{\tau}} \cdot \boldsymbol{\omega}+\left[\left(\overline{\boldsymbol{\tau}} \cdot \overline{\mathbf{p}}_{d}\right) \mathbf{n} \cdot \mathbf{u}\right]_{, \bar{s}} \cdot
$$

Because $\mathbf{u}$ vanishes identically on $\partial \omega \backslash \partial \omega_{t}$ the integral of the last term on the right may be extended to $\partial \omega$ and is thereby seen to contribute nil to the net working if $\partial \omega$ is smooth, i.e., 
if $\overline{\boldsymbol{\tau}}$ is continuous. The same term generates corner forces in the case of a piecewise smooth edge with a finite number of jumps in $\overline{\boldsymbol{\tau}}$. We thus derive (86) with

$$
\mathbf{f}=\overline{\mathbf{p}}_{r}-\left[\left(\overline{\boldsymbol{\tau}} \cdot \overline{\mathbf{p}}_{d}\right) \mathbf{n}\right]_{, \bar{s}} \quad \text { and } \quad M=-\overline{\boldsymbol{v}} \cdot \overline{\mathbf{p}}_{d}
$$

It is interesting that whereas $\overline{\mathbf{p}}_{r}$ and $\overline{\mathbf{p}}_{d}$ are fixed in a formulation based on the threedimensional dead-load problem (see (83)), $\mathbf{f}$ and $M$ are not fixed because they involve the unknown orientation of the deformed surface $\omega$ at the edge $\partial \omega_{t}$. Said differently, $\dot{\mathbf{f}}$ and $\dot{M}$ do not vanish on $\partial \omega_{t}$ although the variations of $\overline{\mathbf{p}}_{r}$ and $\overline{\mathbf{p}}_{d}$ vanish there.

\subsection{The Explicit Energy for Isotropic Materials}

For isotropic materials we have the well-known representation

$$
\mathcal{C}(\mathbf{0})[\mathbf{A}]=\lambda(\operatorname{tr} \mathbf{A}) \mathbf{I}+2 \mu \operatorname{Sym} \mathbf{A},
$$

where $\lambda$ and $\mu$ are the classical Lamé moduli, satisfying the inequalities $3 \lambda+2 \mu>0$ and $\mu>0$ associated with the positivity of $\mathcal{C}(\mathbf{0})$. This furnishes

$$
\{\mathcal{C}(\mathbf{0})[\mathbf{v} \otimes \mathbf{N}]\} \mathbf{N}=(\lambda+2 \mu) v \mathbf{N}+2 \mu \mathbf{1} \mathbf{v}
$$

for any vector $\mathbf{v}$, where $v=\mathbf{v} \cdot \mathbf{N}$. Using this in (73), we derive

$$
\begin{aligned}
& \mathbf{N} \cdot \mathbf{R}^{t} \overline{\mathbf{g}}=-(\lambda+2 \mu)^{-1}\left\{\lambda \operatorname{tr}\left[\mathbf{R}^{t} \nabla \overline{\mathbf{d}}+\mathbf{B}\right]+2 \mu \mathbf{N} \cdot\left(\operatorname{Sym}\left[\mathbf{R}^{t} \nabla \overline{\mathbf{d}}+\mathbf{B}\right]\right) \mathbf{N}\right\} \quad \text { and } \\
& \mathbf{1}\left(\mathbf{R}^{t} \overline{\mathbf{g}}\right)=-2 \mathbf{1}\left(\operatorname{Sym}\left[\mathbf{R}^{t} \nabla \overline{\mathbf{d}}+\mathbf{B}\right]\right) \mathbf{N},
\end{aligned}
$$

which in turn generate $\mathbf{R}^{t} \overline{\mathbf{g}}=\mathbf{1}\left(\mathbf{R}^{t} \overline{\mathbf{g}}\right)+\left(\mathbf{N} \cdot \mathbf{R}^{t} \overline{\mathbf{g}}\right) \mathbf{N}$ for use in (52) and (81).

From (69) the term $\mathbf{R}^{t} \nabla \overline{\mathbf{d}}$ is seen to involve [18]

$$
\mathbf{R}^{t} \nabla \mathbf{n}=\kappa, \quad \text { with } \kappa=-(\nabla \mathbf{r})^{t} \mathbf{b}(\nabla \mathbf{r}),
$$

where $\mathbf{b}$ is the curvature tensor on the deformed surface. In terms of surface coordinates,

$$
\boldsymbol{\kappa}=-b_{\alpha \beta} \mathbf{A}^{\alpha} \otimes \mathbf{A}^{\beta} ; \quad b_{\alpha \beta}=\mathbf{n} \cdot \mathbf{r}_{, \alpha \beta} .
$$

From $\mathbf{R}^{t} \mathbf{n}=\mathbf{N}$ it then follows that

$$
\mathbf{R}^{t} \nabla \overline{\mathbf{d}}+\mathbf{B}=-\boldsymbol{\rho}+\mathbf{N} \otimes \nabla \varphi \quad \text { and } \quad \mathbf{R}^{t} \overline{\mathbf{g}}=-\frac{\lambda}{\lambda+2 \mu}(\operatorname{tr} \boldsymbol{\rho}) \mathbf{N}-\nabla \varphi,
$$

where

$$
\rho=-(\kappa+\mathbf{B}),
$$

which is given in terms of components by Koiter's expression [1, 2]

$$
\boldsymbol{\rho}=\rho_{\alpha \beta} \mathbf{A}^{\alpha} \otimes \mathbf{A}^{\beta} \quad \text { with } \rho_{\alpha \beta}=b_{\alpha \beta}-B_{\alpha \beta} .
$$

This combines with (52) and (92) to give

$$
\mathbf{R}^{t} \mathbf{K}=\rho-\frac{\lambda}{\lambda+2 \mu}(\operatorname{tr} \boldsymbol{\rho}) \mathbf{N} \otimes \mathbf{N}+\mathbf{N} \otimes \nabla \varphi-\nabla \varphi \otimes \mathbf{N}
$$


and (81) then gives the classical bending energy

$$
\mathcal{C}(\mathbf{0})\left[\mathbf{R}^{t} \mathbf{K}\right] \cdot \mathbf{R}^{t} \mathbf{K}=\frac{2 \lambda \mu}{\lambda+2 \mu}(\operatorname{tr} \boldsymbol{\rho})^{2}+2 \mu|\boldsymbol{\rho}|^{2},
$$

in precise agreement with the result obtained by the method of gamma convergence [22] or asymptotic analysis [17].

To reduce the stretching term we invoke $(61)_{1}$ and (63), obtaining

$$
\mathbf{E}=\boldsymbol{\epsilon}-\frac{\lambda}{\lambda+2 \mu}(\operatorname{tr} \boldsymbol{\epsilon}) \mathbf{N} \otimes \mathbf{N} \quad \text { and } \quad \mathcal{C}(\mathbf{0})[\mathbf{E}] \cdot \mathbf{E}=\frac{2 \lambda \mu}{\lambda+2 \mu}(\operatorname{tr} \boldsymbol{\epsilon})^{2}+2 \mu|\boldsymbol{\epsilon}|^{2},
$$

apart from errors of order $o(h)$ and $o\left(h^{2}\right)$, respectively, arriving finally at Koiter's energy (1), in which $\boldsymbol{\epsilon}=O(h)$ and $\mathcal{D}=\mathcal{D}^{\alpha \beta \gamma \delta} \mathbf{A}_{\alpha} \otimes \mathbf{A}_{\beta} \otimes \mathbf{A}_{\gamma} \otimes \mathbf{A}_{\delta}$, with

$$
\mathcal{D}^{\alpha \beta \gamma \delta}=\frac{2 \lambda \mu}{\lambda+2 \mu} A^{\alpha \beta} A^{\gamma \delta}+\mu\left(A^{\alpha \gamma} A^{\beta \delta}+A^{\alpha \delta} A^{\beta \gamma}\right) .
$$

Acknowledgement This note was inspired by a course on Shell Theory delivered by the writer at the University of California.

\section{References}

1. Ciarlet, P.G.: An introduction to differential geometry with applications to elasticity. J. Elast. 78-79, 3-201 (2005)

2. Koiter, W.T.: On the nonlinear theory of thin elastic shells. Proc. K. Ned. Akad. Wet. B 69, 1-54 (1966)

3. Koiter, W.T.: A consistent first approximation in the general theory of thin elastic shells. In: Koiter, W.T. (ed.) Proc. IUTAM Symposium on the Theory of Thin Elastic Shells, Delft, pp. 12-33. North-Holland, Amsterdam (1960)

4. Steigmann, D.J.: Extension of Koiter's linear shell theory to materials exhibiting arbitrary symmetry. Int. J. Eng. Sci. 51, 216-232 (2012)

5. Ball, J.M.: Convexity conditions and existence theorems in nonlinear elasticity. Arch. Ration. Mech. Anal. 63, 337-403 (1977)

6. Healey, T.J., Rosakis, P.: Unbounded branches of classical injective solutions to the forced displacement problem in nonlinear elastostatics. J. Elast. 49, 65-78 (1997)

7. Le Dret, H., Raoult, A.: The nonlinear membrane model as a variational limit of nonlinear threedimensional elasticity. J. Math. Pures Appl. 75, 551-580 (1995)

8. Dacarogna, B.: Direct Methods in the Calculus of Variations. Springer, Berlin (1989)

9. Pipkin, A.C.: The relaxed energy density for isotropic elastic membranes. IMA J. Appl. Math. 36, 85-99 (1986)

10. Hilgers, M.G., Pipkin, A.C.: Bending energy of highly elastic membranes. Q. Appl. Math. 50, 389-400 (1992)

11. Hilgers, M.G., Pipkin, A.C.: Bending energy of highly elastic membranes II. Q. Appl. Math. 54, 307-316 (1996)

12. Steigmann, D.J.: Applications of polyconvexity and strong ellipticity to nonlinear elasticity and elastic plate theory. In: Schröder, J., Neff, P. (eds.) CISM Course on Applications of Poly-, Quasi-, and RankOne Convexity in Applied Mechanics, vol. 516, pp. 265-299. Springer, Vienna (2010)

13. Hilgers, M.G., Pipkin, A.C.: The Graves condition for variational problems of arbitrary order. IMA J. Appl. Math. 48, 265-269 (1992)

14. Ciarlet, P.G.: Plates and Junctions in Elastic Multi-structures: An Asymptotic Analysis. Springer, Berlin (1990)

15. Miara, B., Lods, V.: Nonlinearly elastic shell models I. The membrane model. Arch. Ration. Mech. Anal. 142, 331-353 (1998)

16. Miara, B.: Nonlinearly elastic shells II. The flexural model. Arch. Ration. Mech. Anal. 142, 355-374 (1998) 
17. Ciarlet, P.G., Roquefort, A.: Justification d'un modèle bi-dimensionnel non linéaire de coque analogue à celui de W.T Koiter. C. R. Acad. Sci., Sér. I 331, 411-416 (2000)

18. Steigmann, D.J.: Thin-plate theory for large elastic deformations. Int. J. Non-Linear Mech. 42, 233-240 (2007)

19. Koiter, W.T.: Foundations and basic equations of shell theory. A survey of recent progress. In: Niordson, F.I. (ed.) Proc. IUTAM Symposium on the Theory of Thin Shells, Copenhagen, pp. 93-105. Springer, Berlin (1968)

20. Fox, D.D., Raoult, A., Simo, J.C.: A justification of nonlinear properly invariant plate theories. Arch. Ration. Mech. Anal. 124, 157-199 (1993)

21. Pietraszkiewicz, W.: Geometrically nonlinear theories of thin elastic shells. Adv. Mech. 12, 52-130 (1989)

22. Friesecke, G., James, R.D., Mora, M.G., Müller, S.: Derivation of nonlinear bending theory for shells from three-dimensional elasticity by Gamma-convergence. C. R. Acad. Sci., Sér. I 336, 697-702 (2003) 